\title{
A ‘RIEMANN HYPOTHESIS” FOR TRIANGULABLE MANIFOLDS
}

\author{
K. S. SARKARIA
}

\begin{abstract}
Given a triangulable manifold we show how to find a triangulation whose characteristic polynomial has roots which are either real or on the line $\operatorname{Re} z=1 / 2$.
\end{abstract}

If $K$ is a (finite) simplicial complex, then $f_{K}(z)$ will denote the polynomial $\chi / 2-f_{0}(K) \cdot z+f_{1}(K) \cdot z^{2}-\cdots$; here $\chi$ is the Euler characteristic of the underlying space $M=|K|$ and $f_{i}(K)$ is the number of $i$-simplices in $K$.

THEOREM. If $M$ is any closed triangulable manifold, then it admits a triangulation $K$ for which all the nonreal zeros of $f_{K}(z)$ lie on the line $\operatorname{Re} z=1 / 2$.

Proof. If $L$ is any triangulation of $M^{m}$, then one has the functional equation $f_{L}(z)=(-1)^{m+1} f_{L}(1-z)$. (This fact is well known and is a concise way of writing the Dehn-Sommerville equations (see e.g. [1, p. 101]): it was observed by Klee [2] that these equations hold if the link of each $i$-simplex of $L$ has the same Euler characteristic as an $(m-i-1)$-dimensional sphere, e.g. if $L$ triangulates a closed $m$-manifold.) So the roots of $f_{L}(z)$ are symmetrically situated about the real axis and the line $\operatorname{Re} z=1 / 2$.

For each integer $q \geqslant 0$ we construct a simplicial complex $L_{q}$ as follows: $L_{0}=L$ is any triangulation of $M^{m}$ and $L_{q+1}$ is obtained by deriving an $m$-simplex of $L_{q}$. We note that

$$
\begin{aligned}
f_{L_{q}}(z)= & f_{L}(z)-q z+q(m+1) z^{2}-q\left(\begin{array}{c}
m+1 \\
2
\end{array}\right) z^{3}+\cdots \\
& +(-1)^{m+1} q\left(\begin{array}{c}
m+1 \\
m
\end{array}\right) z^{m+1}-(-1)^{m+1} q z^{m+1} \\
= & f_{L}(z)-q z(1-z)^{m+1}-(-1)^{m+1} q z^{m+1}(1-z) .
\end{aligned}
$$

We assert that for all $q$ sufficiently big $K=L_{q}$ is a triangulation of $M^{m}$ such that $f_{K}(z)$ has distinct roots of which all but 2 lie on the line $\operatorname{Re} z=1 / 2$. It is clear that the remaining 2 roots must then be equal to $1 / 2 \pm \kappa$ for some $\kappa>0$; if $\chi=0$ these exceptional roots are obviously 0 and 1 .

Note that $f_{K}(1-z)=(-1)^{m+1} f_{K}(z)$ and $f_{K}(\bar{z})=\overline{f_{K}(z)}$ imply that for $m$ odd (resp. $m$ even) $f_{K}(z)$ takes real (resp. purely imaginary) values on the line $\operatorname{Re} z=1 / 2$; the same is also true for the degree $m+1$ polynomial

$$
-z(1-z)^{m+1}-(-1)^{m+1} z^{m+1}(1-z)=q^{-1} f_{K}(z)-q^{-1} \cdot f_{L}(z) .
$$

Received by the editors September 3, 1982 and, in revised form, June 6, 1983.

1980 Mathematics Subject Classification. Primary 57Q15; Secondary 52A40, 05C15.

(C) 1984 American Mathematical Society $0002-9939 / 84 \$ 1.00+\$ .25$ per page 
Next we observe that the $m-1$ roots of $-z(1-z)^{m+1}-(-1)^{m+1} z^{m+1}(1-z)$ other than 0 and 1 satisfy $|z /(1-z)|=1$, i.e. lie on the line $\operatorname{Re} z=1 / 2$. So for $q$ big the neighbouring polynomial $q^{-1} f_{K}(z)$ must also have $m-1$ roots on the line $\operatorname{Re} z=1 / 2$. Q.E.D.

REMARK. Let $L$ be a triangulation of $M^{m}$ and let $C(q, m+1), q \geqslant m+2$, be a cyclic triangulation (see e.g. [1, p. 82]) of the sphere $S^{m}$. By omitting an $m$-simplex each from $L$ and $C(q, m+1)$ and then identifying their boundaries, one gets a triangulation $L^{q}$ of $M^{m}$. One can verify (using equation (13) on p. 172 of [1] to examine the roots of the polynomial of $C(q, m+1)$ ) that if $m \geqslant 5$ and $q$ is sufficiently big, then $f_{L^{4}}(z)$ has some roots which are neither real nor on the line $\operatorname{Re} z=1 / 2$.

The "Riemann hypothesis" considered above is related to the lower and upper bound conjectures for manifolds and is amongst the problems posed in $\S 6$ of [3].

I am grateful to the referee for pointing out a mistake in the original version of this paper.

\section{REFERENCES}

1. P. McMullen and G. C. Shepard, Convex polytopes and the upper bound conjecture, Cambridge Univ. Press, London and New York, 1971.

2. V. Klee, A combinatorial analogue of Poincaré's duality theorem, Canad. J. Math. 16 (1964), 517-531.

3. K. S. Sarkaria, On neighbourly triangulations, Trans. Amer. Math. Soc. 277 (1983), 213-239.

213, 16A, Chandigarh 160016, India 